\title{
Chip-based Frequency Comb with Microwave Repetition Rate
}

\author{
Jiang Li, Hansuek Lee, Tong Chen, Kerry Vahala \\ Thomas J. Watson, Sr., Laboratory of Applied Physics, California Institute of Technology, \\ 1200 E. California Boulevard, Pasadena, CA 91125, USA \\ vahala@caltech.edu
}

\begin{abstract}
A silicon-chip microcomb accessing the important microwave-rate FSR range is reported. A broadband microcomb with rep rate $33 \mathrm{GHz}$ and span $66 \mathrm{THz}$ is shown with pump power of $200 \mathrm{~mW}$. Microcombs with rep rates ranging from $132 \mathrm{GHz}$ to $2.6 \mathrm{GHz}$ are demonstrated. OCIS codes: (190.4380) Four-wave mixing; (140.3945) Microcavities; (190.4390) Integrated optics.
\end{abstract}

There is currently intense interest in microcombs and there have been demonstrations using silica micro-toroids [1], $\mathrm{CaF}_{2}$ diamond-milled rods [2,3], silicon-nitride rings on silicon [4], high-index silica rings on silicon [5], and in fiber Fabry-Perots [6]. One priority in this subject is the attainment of microwave-rate free-spectral-range (FSR) in a chip-based platform so as to enable self-referencing [7]. However, there are presently no on-chip micro-combs having repetition rate less than $80 \mathrm{GHz}$. Moreover, the following scaling of threshold power with FSR makes it challenging to reduce FSR without increasing threshold power.

$$
P_{t h}=\frac{(1+K)^{3}}{8 K} \frac{n}{n_{2}} \frac{\omega}{\Delta \omega} \frac{A}{Q^{2}}
$$

where $\mathrm{K}$ is the normalized external coupling rate, $n_{2}(n)$ is the nonlinear index (refractive index), $\Delta \omega(\omega)$ is the freespectral-range (optical frequency), $A$ is the mode area, and $Q$ is the resonator optical $\mathrm{Q}$ factor. In this work, we report a silicon-chip-based microcomb that accesses the important microwave-rate FSR range. At the same time, the device also achieves a low threshold turn-on power typically in the range of a few $\mathrm{mW}$.

Improving the optical Q factor is an extremely effective way to offset the impact of reduced FSR $(\Delta \omega)$ on threshold (see Eq. 1). Higher Q creates larger resonant build-up so that a given coupled power creates a greater Kerr-induced four-wave-mixing (FWM) of signal and idler waves. It also reduces oscillation threshold since optical loss is reduced. The microcomb of this work uses a new chip-based resonator that provides $\mathrm{Q}$ factors as high as 870 million [8]. Significantly, these devices do not require silica reflow [9], which becomes problematic at diameters approaching $1 \mathrm{~mm}$. Rather, only conventional semiconductor processing methods are necessary (lithography and wet etch) so that ultra-high-Q devices featuring FSR's of a few GHz to hundreds of GHz are possible.

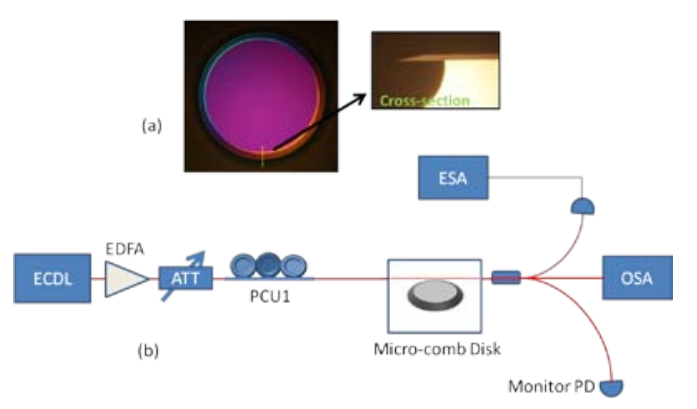

Fig. 1. (a) Top and side view of the wedge disk resonator. (b) Schematic of typical experimental setup

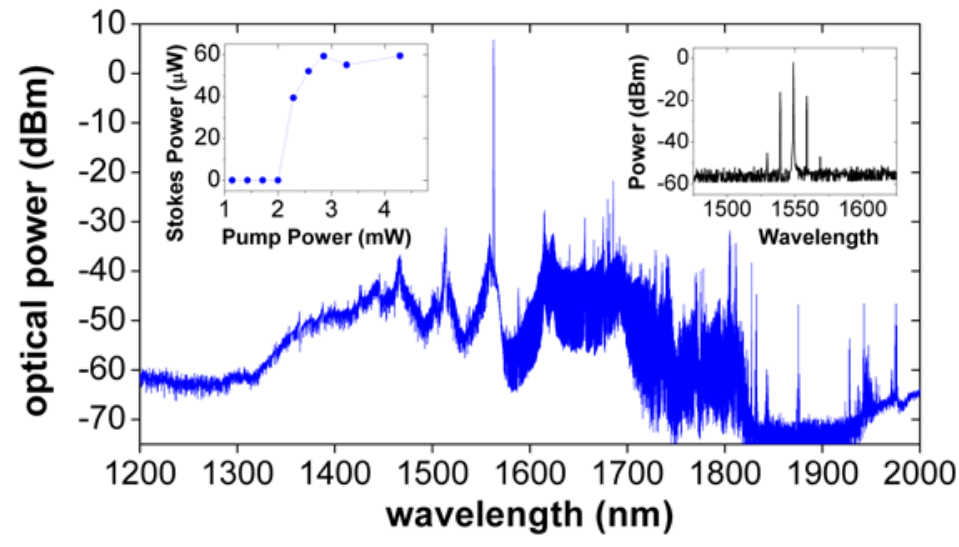

Fig. 2. Main panel: Broadband comb spectrum with $66 \mathrm{THz}$ span and $33 \mathrm{GHz}$ rep rate. Left Inset: First FWM sideband power vs pump power; Right inset: comb spectrum just above threshold.

In a typical experimental setup (see Fig. 1), the amplified pump laser is coupled to the disk resonator using a tapered fiber $[10,11]$ and is thermally locked within the cavity resonance [12]. When the coupled, pump power exceeds threshold, FWM lines turn-on very abruptly (see Fig. 2. insets). A threshold less than $2.3 \mathrm{~mW}$ is measured for a $2 \mathrm{~mm}$, wedge disk having an intrinsic Q of 300 million. The right inset of Fig. 2 shows that the first generated FWM 
lines are typically multiple cavity FSR away from the pump, where the dispersion is compensated by nonlinear phase shift and the parametric gain is maximized. The main panel of figure 2 shows that with coupled pump power increased to $200 \mathrm{~mW}$ a broadband comb spectrum is generated with $66 \mathrm{THz}$ span and $33 \mathrm{GHz}$ rep rate. Furthermore, with these devices, we are able to generate chip-based microcombs with rep rates covering the entire microwave spectrum. Fig. 3 shows demonstrated rates ranging from $132 \mathrm{GHz}$ to $2.6 \mathrm{GHz}$ plotted versus resonator diameter. The $2.6 \mathrm{GHz}$ repetition rate is the smallest demonstrated so far for any microcomb.

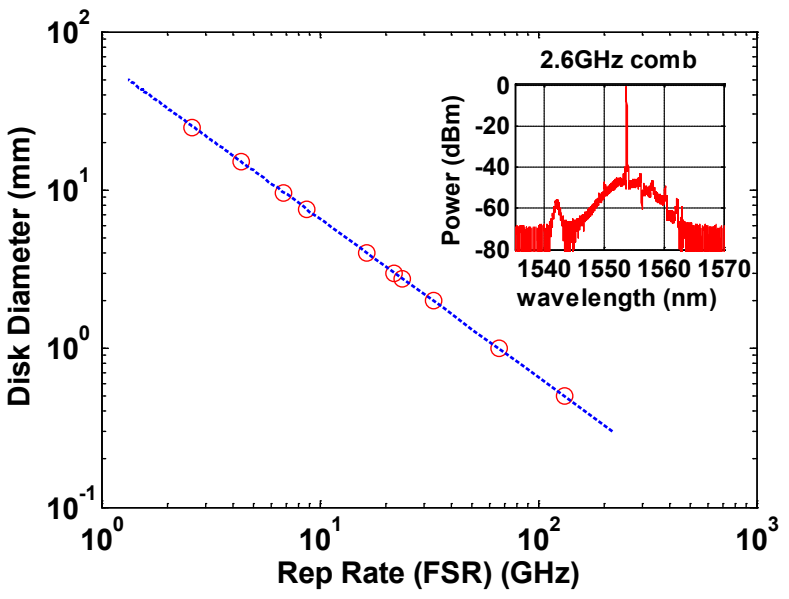

Fig. 3. Demonstrated microcomb rep rates from $132 \mathrm{GHz}$ to $2.6 \mathrm{GHz}$ plotted versus resonator diameter. Inset: An optical spectrum for $2.6 \mathrm{GHz}$ comb.

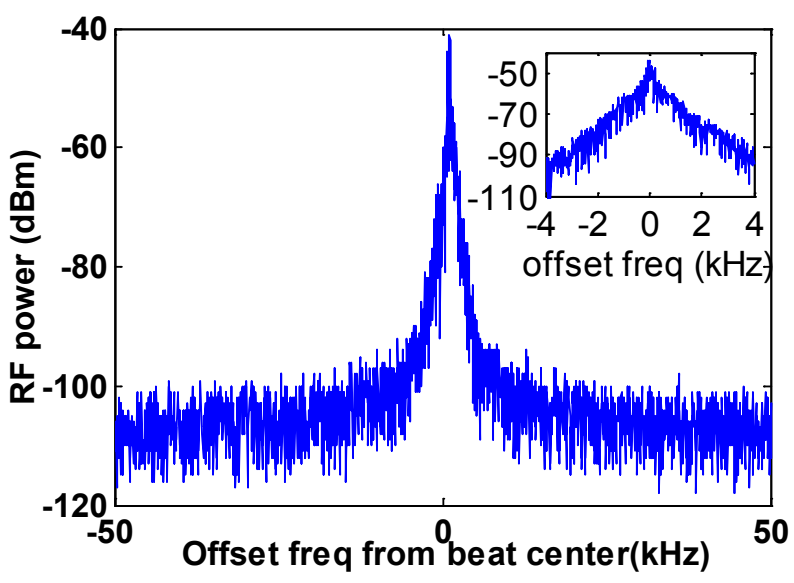

Fig. 4. Microwave beat note spectrum of the $21.9 \mathrm{GHz}$ repetition rate Kerr comb (RBW $300 \mathrm{~Hz}$, span 100kHz). Inset: zoom-in measurement of the beat note, RBW100Hz, span $8 \mathrm{kHz}$

In order to characterize the coherence of this microwave rate Kerr-comb, we directly demodulate the optical comb by detection on a fast photo-receiver with bandwidth $25 \mathrm{GHz}$. The detected electrical spectrum using a $3 \mathrm{~mm}$ disk $(\mathrm{FSR}=21.9 \mathrm{GHz})$ is shown in Fig. 4. Without any external locking, the free-running, mode-locked Kerr comb features a $3 \mathrm{~dB}$ repetition rate linewidth in the 100 s Hertz level, which illustrates the high coherence of the Kerr comb.

In conclusion, a chip-based microcomb at microwave rates from $132 \mathrm{GHz}$ to $2.6 \mathrm{GHz}$ has been demonstrated for the first time. The Kerr comb beat note is easily detected and is highly coherent with a typical free-running beat linewidth at the 100s of Hertz level. The devices also feature milliWatt level threshold.

\section{References}

[1] P. Del'Haye, A. Schliesser, O. Arcizet, T. Wilken, R. Holzwarth and T. J. Kippenberg, “Optical frequency comb generation from a monolithic microresonator," Nature 450, 1214 (2007)

[2] A. A. Savchenkov, A. B. Matsko, V. S. Ilchenko, I.Solomatine, D. Seidel, and L. Maleki, "Tunable Optical Frequency Comb with a Crystalline Whispering Gallery Mode Resonator," Physical Review Letter 101, 093902 (2008)

[3] I. S. Grudinin, N. Yu, and L. Maleki, "Generation of optical frequency combs with a $\mathrm{CaF}_{2}$ resonator," Opt. Lett. 45, 878-880 (2009).

[4] J. S. Levy, A. Gondarenko, M. A. Foster, A. C. Turner-Foster, A. L. Gaeta, and M. Lipson, "CMOS-compatible multiple-wavelength oscillator for on-chip optical interconnects," Nature Photon. 4, 37-40 (2010)

[5] L. Razzari, D. Duchesne, M. Ferrera, R. Morandotti, S. Chu, B. E. Little, and D. J. Moss, "CMOS-compatible integrated optical hyperparametric oscillator," Nature Photon. 4, $41-45$ (2010)

[6] D. Braje, L. Hollberg, and S. Diddams, "Brillouin-enhanced hyperparametric generation of an optical frequency comb in a monolithic highly nonlinear fiber cavity pumped by a cw laser," Phys. Rev. Lett. 102, 193902 (2009)

[7] T. J. Kippenberg, R. Holzwarth, and S. A. Diddams, "Microresonator-Based Optical Frequency Combs," Science 332, 555-559 (2011)

[8] H. Lee, T. Chen, J. Li, O. Painter and K. Vahala, "Ultra-High-Q Micro-Cavity on a Silicon Chip", FIO (2011)

[9] D. K. Armani, T. J. Kippenberg, S. M. Spillane and K. J. Vahala, "Ultra-high-Q toroid microcavity on a chip," Nature, 421, $925-929$ (2003)

[10] M. Cai, O. Painter and K. Vahala, "Observation of critical coupling in a fiber taper to silica-microsphere whispering gallery mode system," Phys. Rev. Lett. 85, 74-77, (2000)

[11] S. M. Spillane, T. J. Kippenberg, O. J. Painter, and K. J. Vahala, "Ideality in a Fiber-Taper-Coupled Microresonator System for Application to Cavity Quantum Electrodynamics," Phys. Rev. Lett. 85, 043902, (2003)

[12] T. Carmon, L. Yang, and K.J. Vahala, "Dynamical thermal behavior and thermal self-stability of microcavities," Optics Express 12, 4742 (2004) 